\title{
Efeitos do Tipo de Poliestireno de Alto Impacto nas Propriedades de Compósitos Termoplásticos com Farinha de Resíduo de Madeira
}

\author{
Wladimir L. Vianna, Carlos A. Correa \\ Programa de Pós-Graduação em Engenharia e Ciência de Materiais, USF \\ Carlos A. Razzino \\ Departamento de Engenharia de Materiais, UFSCar
}

\begin{abstract}
Resumo: A tecnologia dos chamados Compósitos Termoplásticos com Madeira envolve conceitos de compatibilidade e processabilidade e apresenta grandes desafios tecnológicos para a formulação e estabilização da mistura. Devido à baixa estabilidade térmica da celulose, a grande maioria das resinas termoplásticas utilizadas na preparação desses compósitos está restrita às resinas poliolefinicas, estirênicas e ao poli(cloreto de vinila). O Poliestireno de Alto Impacto (HIPS) é um termoplástico bifásico de grande versatilidade de propriedades decorrentes das variações de composição e morfologia das partículas de borracha dispersas na matriz estirênica. Além da sua temperatura relativamente baixa de processamento, estas características tornam o HIPS um termoplástico de grande interesse para aplicações em compósitos com farinha de resíduo de madeira, uma vez que a otimização do balanço de propriedades de rigidez e impacto pode ser alcançada através do controle da morfologia e da formulação do compósito. No presente estudo, "tipos" comerciais de HIPS com diferenças no índice de fluidez e na distribuição do tamanho de partículas foram utilizados na preparação de compósitos termoplásticos com farinha de resíduo de madeira. As propriedades mecânicas dos compósitos obtidos foram discutidas em termos das características morfológicas dos HIPS utilizados e da quantidade de farinha de resíduo de madeira no compósito. Pela simples aplicação da regra das misturas, mostrou-se que devido à baixa densidade do resíduo de madeira, o reforço celulósico poderia ser utilizado eficientemente na substituição de cargas minerais ou fibra de vidro em compósitos termoplásticos com um melhor desempenho em termos de resistência e rigidez específica. A microscopia eletrônica de varredura foi utilizada na análise de superfícies de fratura para ilustrar a dispersão da farinha de madeira na matriz estirênica, a molhabilidade do reforço pela resina e as interações reforço-matriz.
\end{abstract}

Palavras-chave: Compósitos termoplásticos, reforços celulósicos, poliestireno de alto impacto, farinha de madeira, propriedades mecânicas.

\section{The Effects of the High Impact Polystyrene Morphology on the Properties of Wood-Plastic Composites}

\begin{abstract}
The technology of wood-plastics composites includes concepts of compatibility and processability, with important challenges to optimize grade formulations, processing and stabilization of the composite system. Owing to the low thermal stability in wood flour processing, commodities such as polyolefins, styrene and polyvinylchloride represent the large majority of the thermoplastics employed in cellulosic composites. High impact polystyrene (HIPS) is a versatile thermoplastic as a result of variations in composition and morphology of the rubber dispersed phase in the styrenic matrix. In addition to its relatively low processing temperature, such characteristics make HIPS a suitable polymer for wood-plastic composite applications as an optimum stiffness-toughness balance can be achieved by fine control of HIPS morphological parameters and the composite formulation. In the present study, commercial grades of HIPS with different flow index and particle size distribution were used in the preparation of wood-plastic composites. The mechanical properties and the heat distortion temperatures of the composites are discussed in terms of the HIPS characteristics and the filler content in the composite. By simply applying the rule of mixtures, it was shown that owing to their relatively low specific gravity, the wood waste flour might be cost-effective in replacing mineral fillers or glass fibers in plastic composites with a better performance in terms of specific strength and rigidity. Electron microscopy analysis of fractured surfaces was used to illustrate wood flour dispersion, wettability and matrix-filler interactions.
\end{abstract}

Keywords: Composites, cellulosic fillers, HIPS, wood-waste flour, wood-filler interface.

Autor para correspondência: Carlos A. Correa, USF, Rua Alexandre R. Barbosa 45, CEP: 13521-900, Itatiba, SP. E-mail: carlos.correa@saofrancisco.edu.br 


\section{Introdução}

A preparação de compósitos de madeira com polímeros é uma prática antiga, particularmente quanto ao uso de resinas termofixas como uréia-, fenol-, ou melamina-formaldeído e isocianatos na produção de painéis MDF (medium-density fiberboard). Da mesma forma, a utilização de farinha ou fibra de madeira como carga em termoplásticos, também já é conhecida desde a década de 70 pela indústria automobilística, que emprega compósitos de polipropileno com farinha de madeira, conhecidos no mercado, como woodstock ${ }^{\mathbb{R}}$. Esses compósitos são extrudados e laminados em chapas para revestimento interno de portas e porta-malas de veículos em uso corrente. Inúmeros aspectos devem ser observados no processamento de termoplásticos com resíduos de madeira. A umidade e a granulometria devem ser rigidamente controladas, uma vez que esta produz descontinuidades de processo e peças com características inaceitáveis devido à presença de bolhas ou manchas superficiais causadas por processos termooxidativos. Assim, como principal requisito, o resíduo celulósico deve ser pré-secado e zonas de degasagem devem ser utilizadas para remoção da umidade residual e voláteis durante o processamento ${ }^{[1]}$.

A baixa temperatura de degradação da celulose na faixa de 200-220 ${ }^{\circ} \mathrm{C}$ constitui um fator limitante do processo, exceto quando os tempos de residência são minimizados. A exposição do resíduo de madeira à temperaturas acima dessa faixa libera voláteis, provoca descoloração, aparecimento de odor e fragilização do compósito ${ }^{[1-3]}$. Isso tem restringido o uso de termoplásticos em compósitos com madeira às principais resinas comerciais: poliolefinas, plásticos estirênicos e o poli(cloreto de vinila).

O principal benefício da utilização de farinha de madeira em termoplásticos consiste no aumento da rigidez e da temperatura de uso desses materiais embora este ganho seja alcançado a custo de reduções drásticas na tenacidade. $\mathrm{O}$ poliestireno de alto impacto (HIPS) pode ser considerado uma resina de grande interesse para uso em compósitos termoplásticos com madeira uma vez que as suas proprie- dades mecânicas e reológicas estão fortemente relacionadas com a morfologia e distribuição dos tamanhos de partículas de polibutadieno na matriz estirênica ${ }^{[4-6]}$.

Nesse estudo foram utilizados três tipos comerciais de HIPS variando-se o índice de fluidez da resina e a morfologia das partículas de borracha, procurando-se assim avaliar o efeito dessas variáveis no balanço de propriedades de rigidez e tenacidade do compósito.

\section{Experimental}

\section{Materiais}

Os tipos de HIPS utilizados neste trabalho foram fabricados e fornecidos pela empresa BASF S.A. na forma de grânulos sob especificação 476L; 495F e 585K. Estes materiais foram sintetizados por polimerização em massa (476L e 595F) e emulsão (585K) e possuem um teor de borracha na faixa de 8,5 a 9,0\% dispersa na forma de partículas na matriz estirênica. As propriedades desses materiais fornecidas pelo fabricante, são apresentadas na Tabela 1 .

A farinha de resíduo de madeira ( $w w f$ - wood waste flour) foi fornecida pela empresa PinhoPó com especificação granulométrica de $45 \mathrm{mesh}$. As demais propriedades utilizadas para farinha nesse trabalho foram o Módulo de Elasticidade longitudinal em compressão paralela às fibras, $E_{f}=8,5$ GPa e Peso específico a $12 \%$ de Umidade Relativa, $\rho_{\mathrm{f}}=579 \mathrm{~kg} / \mathrm{m}^{3}$. Estes valores foram obtidos na literatura para a madeira Pinus caribea ${ }^{[8]}$.

\section{Metodologia}

Os compósitos de HIPS com a farinha de madeira foram preparados em extrusora de dupla-rosca co-rotacional de bancada marca B\&P Process Equipments Systems. Foram preparadas misturas contendo $20 ; 30$ e $40 \%$ em peso de farinha de madeira. Corpos-de-prova de tração, impacto e Temperatura de Distorção Térmica (HDT) foram moldados em uma injetora modelo 270V da ARBURG.

As propriedades mecânicas foram avaliadas através do ensaio de tração uniaxial (ASTM D638) utilizando-se os corpos-de-prova

Tabela 1. Propriedades dos HIPS puros ${ }^{[7]}$

\begin{tabular}{|c|c|c|c|c|c|c|}
\hline \multirow{2}{*}{\multicolumn{2}{|c|}{ Propriedades }} & \multicolumn{2}{|c|}{ ISO } & \multicolumn{3}{|c|}{ Valores Típicos } \\
\hline & & \multirow{2}{*}{$\begin{array}{c}\text { Norma } \\
\text { ISO-1133 }\end{array}$} & \multirow{2}{*}{$\begin{array}{c}\begin{array}{c}\text { Unidades } \\
\text { S.I. }\end{array} \\
\text { g/10min }\end{array}$} & \multirow{2}{*}{$\begin{array}{c}\text { Polystyrol } \\
\text { 476L }\end{array}$} & \multirow{2}{*}{$\begin{array}{c}\begin{array}{c}\text { Polystyrol } \\
\mathbf{4 9 5 F}\end{array} \\
10\end{array}$} & \multirow{2}{*}{$\begin{array}{c}\begin{array}{c}\text { Polystyrol } \\
\text { 585K }\end{array} \\
5\end{array}$} \\
\hline 1 & Índice de Fluidez & & & & & \\
\hline 2 & Módulo de elasticidade (tração) & ISO-527 & $\mathrm{GPa}$ & 1.9 & 1.9 & 1.9 \\
\hline 3 & Resistência à tração (escoamento) & ISO-527 & $\mathrm{MPa}$ & 24 & 21 & 27 \\
\hline 4 & Alongamento (ruptura) & ISO-527 & $\%$ & 70 & 34 & 13 \\
\hline 5 & Resistência ao impacto CHARPY & ASTM D-256 & $\mathrm{J} / \mathrm{m}$ & 148 & 148 & 90 \\
\hline 6 & $\begin{array}{l}\text { Temperatura de deflexão térmica } \\
\text { (HDT) - ISO-75 - Método (A) }\end{array}$ & ISO-75 & ${ }^{\circ} \mathrm{C}$ & 85 & 83 & 77 \\
\hline 7 & Densidade & ISO-1183 & $\mathrm{kg} / \mathrm{m}^{3}$ & 1050 & 1040 & 1050 \\
\hline
\end{tabular}




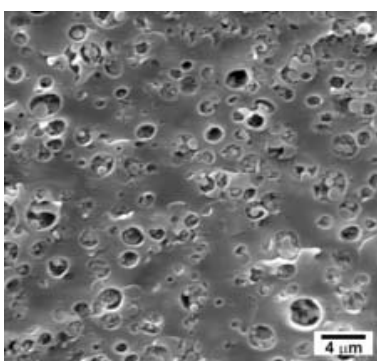

a) $476 \mathrm{~L}$

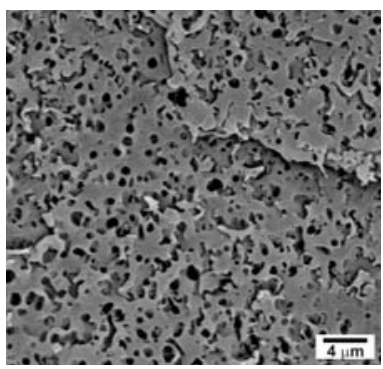

b) $495 \mathrm{~F}$

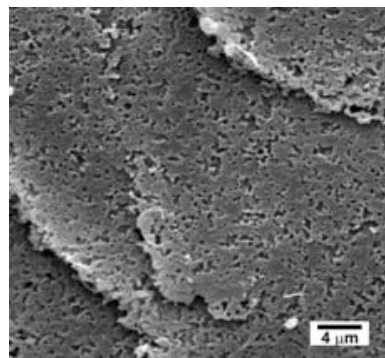

c) $585 \mathrm{~K}$

Figura 1. Microscopia eletrônica de varredura das amostras de HIPS puro após a extração da fase borracha.

moldados por injeção do tipo M-I e barras para ensaio de impacto IZOD (ASTM D-256). Os testes para determinação de HDT foram realizados segundo a norma ISO 75, Método A.

As medidas de distribuição de tamanhos das partículas de borracha foram realizadas através da imersão dos corpos-deprova entalhados em $\mathrm{N}_{2}$ líquido seguido por impacto criogênico. A seguir, as superfícies de fratura do HIPS foram submetidas a um ataque químico com solução sulfocrômica $\left(100 \mathrm{ml} \mathrm{H}_{2} \mathrm{SO}_{4}\right.$; $30 \mathrm{ml} \mathrm{H}_{3} \mathrm{PO}_{4} ; 30 \mathrm{ml} \mathrm{H}_{2} \mathrm{O}$ e $5 \mathrm{~g} \mathrm{CrO}_{3}$ ) a $70{ }^{\circ} \mathrm{C}$ por 3 minutos para remoção da fase borrachosa ${ }^{[6]}$. Após o ataque químico, todas as amostras foram recobertas com um fina camada de carbono em um evaporador à vácuo. As micrografias obtidas por Microscopia Eletrônica de Varredura (MEV) foram analisadas utilizando-se o Software Adobe Photoshop contabilizando-se cerca de 100 medidas de diâmetro de partículas de borracha para cada grade de HIPS analisado.

As análises morfológicas dos materiais puros e dos compósitos foram realizadas utilizando-se um microscópio eletrônico de varredura (MEV) modelo LEO 400 com voltagem de aceleração de $20 \mathrm{kV}$.

\section{Granulometria da farinha de resíduo de madeira (wwf)}

Para a obtenção da distribuição granulométrica da farinha de resíduo de madeira foi utilizado um agitador eletromecânico com 6 peneiras de 8"x2", dispositivo para controle da freqüência de vibrações e relógio automático de $30^{\prime}$. O equipamento foi fabricado segundo a norma ABNT/EB-22 pela Soloeste Aparelhos para Mecânica do Solo Ltda, SP. A distribuição granulométrica foi obtida a partir da distribuição de massa retida do resíduo após 10 min de vibração.

\section{Resultados e Discussão}

\section{Caracterização morfológica do HIPS}

As principais características morfológicas dos tipos de poliestireno de alto impacto empregados nesse estudo são apresentados na Figura 1 para as imagens obtidas por MEV a partir

Tabela 2. Diâmetros médios das partículas de borracha dos HIPS

\begin{tabular}{lccc}
\hline & $\mathbf{4 7 6 L}$ & $\mathbf{4 9 5} \mathbf{F}$ & $\mathbf{5 8 5 K}$ \\
\hline Diâmetro médio $(\mu \mathrm{m})$ & 1,25 & 0,69 & 0,30 \\
Desvio Padrão & 0,65 & 0,23 & 0,16 \\
Erro 95\% confiança & 0,15 & 0,05 & 0,03 \\
\hline
\end{tabular}

das superfícies criofraturadas e atacadas quimicamente. A extração da fase borracha permite medidas do tamanho das cavidades e uma avaliação quantitativa da distribuição de tamanhos das partículas conforme apresentado na Figura 2 para os três tipos diferentes de HIPS obtidos através de análise digital de imagens. Os valores médios de tamanho de partículas para cada grade são resumidos na Tabela 2

Observa-se na Tabela 2 que o HIPS 476L obtido pelo processo em massa apresenta o maior tamanho de partícula se comparados aos demais tipos, particularmente o HIPS 585K com tamanho médio e distribuição de tamanho de partículas, característico dos tipos obtidos por emulsão (Baixo tamanho médio e distribuição estreita do espectro de tamanho de partículas).

\section{Fração volumétrica de partículas de borracha (FVPB) dos HIPS}

A FVPB foi calculada para cada tipo de HIPS, utilizandose nos cálculos a relação entre as áreas ocupadas pelas partículas para uma determinada área de matriz. Considerou-se a fração em área $\left(\phi_{\mathrm{A}}\right)$ equivalente à fração volumétrica real das partículas com uma boa aproximação para espessura desprezível, i.e. uma superfície matemática (espessura, $t \rightarrow 0$ ), a partir da seguinte relação matemática:

$$
\Phi=\frac{4 \bar{R}}{4 \bar{R}+3 t} \Phi_{\mathrm{A}}
$$

onde $\phi=$ Fração volumétrica real das partículas na matriz; $\Phi_{\mathrm{A}}=$ fração aparente de partículas ou observada na micrografia; e $\bar{R}=$ Raio médio real das partículas e $\mathrm{t}=$ espessura do filme.

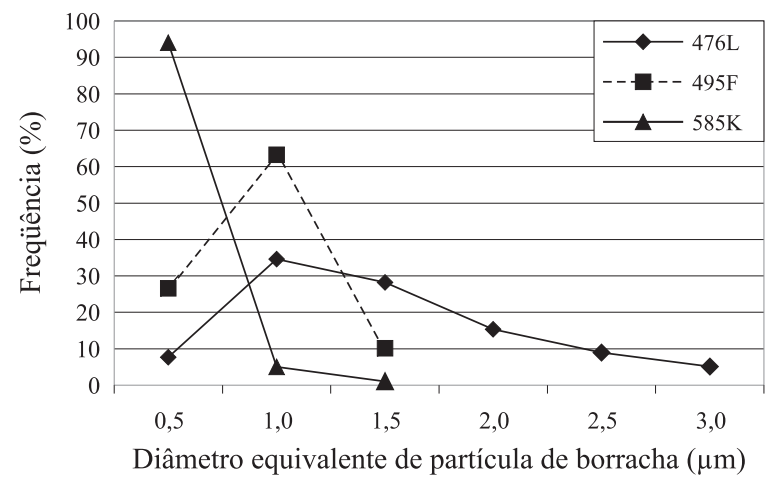

Figura 2. Distribuição de tamanhos de partículas de borracha para três tipos de HIPS 
Tabela 3. Relação entre FVPB e PS (Matriz) nos tipos de HIPS

HIPS 476L HIPS 495F HIPS 585K

\begin{tabular}{lccc}
\hline \% PS (Matriz) & 67,6 & 75,3 & 89,5 \\
$\%$ FVPB & 32,4 & 24,7 & 10,5 \\
$\%$ Total & 100 & 100 & 100 \\
\hline
\end{tabular}

A equação mencionada tem sido utilizada na análise de imagens de filmes de espessura finita observados por microscopia eletrônica de transmissão e micrografias obtidas por MEV com aproximação para espessura $\mathrm{t}=0$ (superfícies criofraturadas), considerando-se que as distribuições de tamanho de partículas como distribuições monomodais ${ }^{[9-11]}$. Observando-se os tamanhos médios e os respectivos desvios padrões dos HIPS apresentados na Tabela 2, esta aproximação pode ser considerada bastante razoável.

$\mathrm{Na}$ Tabela 3 são apresentados os dados com as porcentagens de PS matriz e FVPB para cada HIPS utilizado neste trabalho.

Observa-se que a fração volumétrica ocupada pelas partículas de borracha (FVPB) é maior no 476L, uma vez que a maior quantidade de oclusões observadas neste tipo de HIPS aumenta consideravelmente o tamanho das partículas de borracha e consequentemente o volume ocupado por estas na matriz. Os dados obtidos para FVPB nos HIPS apresentado na Tabela 3 mostram que estas medidas variam de $10 \%$ a $32 \%$ nos HIPS estudados.

\section{Classificação granulométrica da farinha de resíduo de madeira (wwf)}

Os dados obtidos para classificação granulométrica da farinha de resíduo de madeira são apresentados na Figura 3 conforme resultados obtidos a partir do ensaio de penei-

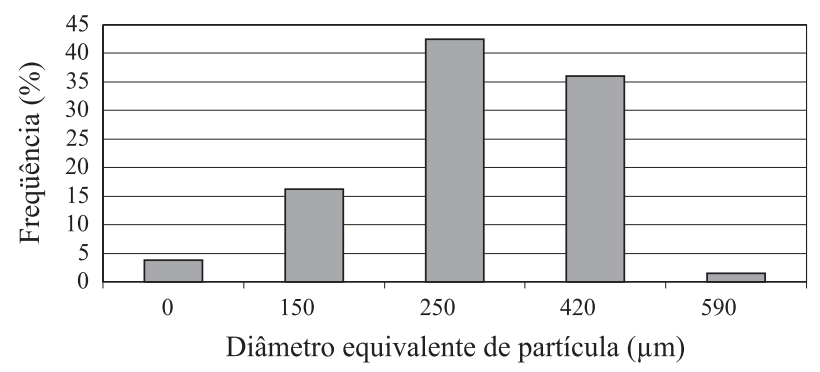

Figura 3. Distribuição de tamanho de partículas da farinha de resíduo de madeira segundo a classificação granulométrica por peneiramento.

Tabela 4. Dimensões das peneiras segundo a malha ASTM

\begin{tabular}{cc}
\hline Malha & Dimensão $(\boldsymbol{\mu m})$ \\
\hline 30 & 590 \\
40 & 420 \\
60 & 250 \\
100 & 150 \\
Fundo & - \\
\hline
\end{tabular}

ramento descrito anteriormente para a série de peneiras da Tabela 4.

As malhas que apresentaram a maior porcentagem de wwf retida foram as malhas $60(250 \mu \mathrm{m})$ e $40(420 \mu \mathrm{m})$, cuja especificação está em concordância com os dados do fornecedor.

\section{Características dimensionais do resíduo de farinha de madeira (wwf)}

A caracterização do resíduo foi realizada utilizando-se micrografias obtidas por MEV e analisadas com um software para análise digital de imagens. Foram determinados os diâmetros equivalentes de cerca de 300 partículas. Embora as partículas apresentassem perfis alongados, estes foram representados para geometrias circulares e os diâmetros equivalentes dos círculos foram determinados e tabulados (Tabela 5).

A razão de aspecto média $(\lambda)$ foi definida como base na relação entre o diâmetro máximo $\left(\mathrm{D}_{\max }\right)$ e mínimo $\left(\mathrm{D}_{\min }\right)$ de cada partícula obtidos no analisador de imagens conforme ilustrado na Figura 4.

$$
\bar{\lambda}=\sum_{i=1}^{n}\left(\frac{D_{\max }}{D_{\min }}\right)_{i}
$$

Com base nos resultados obtidos para a razão de aspecto média, o resíduo de farinha de madeira pode ser considerado como fibras curtas segundo definição da literatura, ou seja, razão de aspecto, $\lambda<10^{[12]}$.

Tabela 5. Características dimensionais da Farinha de Resíduo de Madeira

\begin{tabular}{lc}
\hline Diâmetro equivalente médio $(\mu \mathrm{m})$ & $635 \pm 133$ \\
Diâmetro equivalente máximo $(\mu \mathrm{m})$ & 964 \\
Diâmetro equivalente mínimo $(\mu \mathrm{m})$ & 287 \\
Razão de aspecto média & $2,16 \pm 0,86$ \\
\hline
\end{tabular}

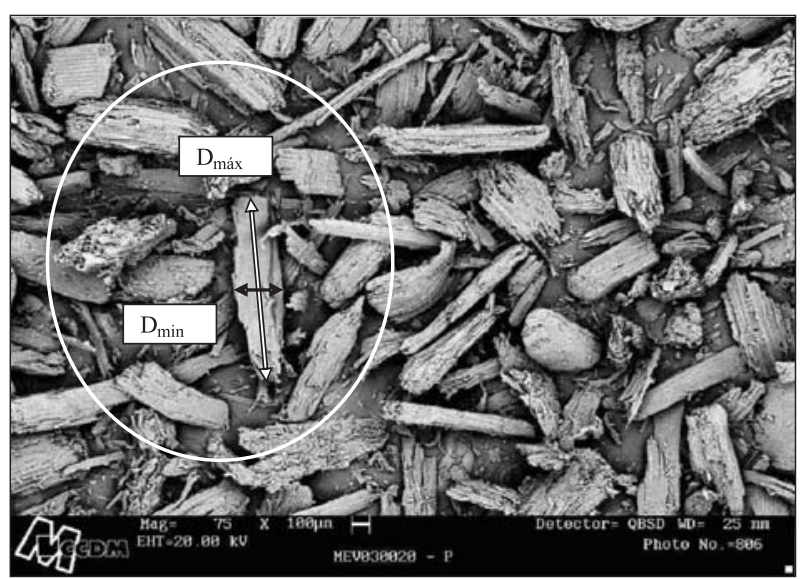

Figura 4. Características geométricas da farinha de resíduo de madeira através de fotomicrografia obtida por MEV. 


\section{Propriedades Mecânicas}

Os efeitos da morfologia nas propriedades mecânicas do HIPS tem sido extensivamente discutidos na literatura ${ }^{[4-6]}$. Neste trabalho, os principais efeitos observados foram as diferenças de fluidez do polímero e da FVPB na rigidez, resistência à tração, alongamento na ruptura e resistência ao impacto desses materiais compósitos. Dentre os materiais puros, o HIPS 476L, com tamanho médio de partículas superior aos demais, apresentou níveis de tensão de escoamento menor e tenacidade sob impacto mais elevado. O tamanho de partícula de borracha não possui influência significativa sobre a rigidez dos materiais puros embora o HIPS com maior tamanho de partícula apresentou um alongamento na ruptura superior aos demais tipos estudados.

Nos compósitos, os principais ganhos de propriedade estiveram relacionados ao aumento de rigidez e temperatura de distorção térmica. $\mathrm{O}$ aumento no módulo elástico chegou a $150 \%$ para a composição com $40 \%$ de madeira no compósito enquanto incrementos de $10^{\circ} \mathrm{C}$ foram observados no HDT dos compósitos. Todavia, a resistência ao impacto para todos os compósitos foi reduzida a $20 \%$ do valor do HIPS puro independente do tipo de morfologia.

\section{Determinação das Frações Volumétricas}

As frações volumétricas da fibra, $V_{f}$ (farinha de madeira) e da matriz $V_{m}$ (HIPS) podem ser obtidas a partir das propriedades intrínsecas desses materiais conforme indicado abaixo para um compósito com $20 \%$ de farinha de madeira.

$$
V_{f}=\frac{m_{f} / \rho_{f}}{m_{f} / \rho_{f}+m_{m} / \rho_{m}}=\frac{0,2 / 579}{0,2 / 579+0,8 / 1050}=0,312
$$

$V_{f}=0,312$ ou $31,2 \%$ (Fração volumétrica do resíduo de farinha de madeira - wwf)

$$
V_{m}=\frac{m_{m} / \rho_{m}}{m_{m} / \rho_{m}+m_{f} / \rho_{f}}=\frac{0,8 / 1050}{0,8 / 1050+0,2 / 579}=0,688
$$

$V_{m}=0,688$ ou $68,8 \%$ (Fração volumétrica da matriz - HIPS)

\section{Cálculo do Módulo de Elasticidade Teórico}

Aplicando-se a "regra das misturas" para compósitos reforçadas com fibras descontínuas e aleatoriamente orientadas (fibras curtas), além dos valores discriminados anteriormente, têm-se para uma mistura de HIPS/20wwf:

$$
\mathrm{E}_{c}=K \cdot \mathrm{E}_{f} \cdot V_{f}+E_{m} \cdot V_{m}
$$

onde

$E_{\mathrm{c}}=$ Módulo de Elasticidade Teórico do Compósito;

$E_{f}=8,5 \mathrm{GPa}$ (Módulo de Elasticidade longitudinal, obtido no ensaio de compressão paralela às fibras, da farinha de madeira Pinus caribea;

\begin{tabular}{|c|c|c|c|c|}
\hline $\begin{array}{c}\text { Ec } \\
(\mathbf{G P a})\end{array}$ & 1,9 & 4,0 & 4,8 & 5,5 \\
\hline
\end{tabular}

Tabela 6. Módulo de Elasticidade Teórico - Limite superior

\section{HIPS HIPS/20wwf HIPS/30wwf HIPS/40wwf}

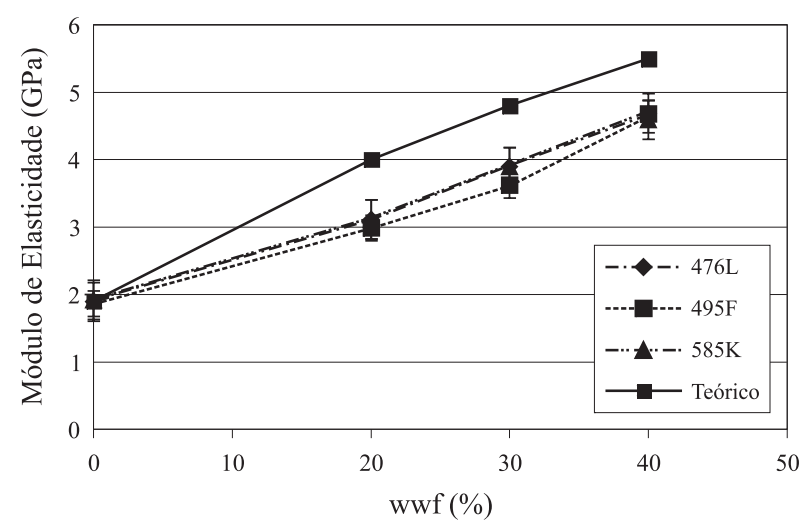

Figura 5. Efeito da concentração de madeira e do tipo de HIPS no Módulo de Elasticidade em Tração com Intervalo de Confiança de 95\%.

$E_{m}=1,9 \mathrm{GPa}$ (Módulo de Elasticidade do HIPS, Tabela 1); $\mathrm{K}=$ Fator eficiência de reforço.

Considerando-se a eficiência de reforço para um material ideal $(\mathrm{K}=1,0)$, pode-se calcular o máximo limite superior do módulo de elasticidade teórico. Ilustrando-se como exemplo o cálculo para a composição 476L/20wwf.

$V_{f}=0,312$ ou $31,2 \%$ (Fração Volumétrica da fibra - wwf); $V_{m}=0,688$ ou $68,8 \%$ (Fração Volumétrica da matriz - HIPS); $V_{f}+V_{m}=1,0$ (Ausência de vazios)

$$
\mathrm{E}_{\mathrm{c}}=1,0 \times 8,5 \times 0,312+1,9 \times 0,688=4,0 \mathrm{GPa}
$$

Os cálculos para as demais composições são apresentados na Tabela 6.

Estes valores são apresentados no gráfico da Figura 5 onde são comparados aos resultados obtidos em laboratório para todas as composições estudadas, caracterizando assim o limite máximo superior dos módulos elásticos para eficiência máxima de reforço $(K=1,0)$ e ausência de vazios, ou seja, sistema híbrido ideal.

$\mathrm{Na}$ literatura, também são observados aumentos nos módulos elásticos nos estudos de HIPS reciclado com $30 \%$ de farinha de madeira Pinus elliotti ${ }^{[13]}$, de PP com $40 \%$ de farinha de madeira ${ }^{[9]}$ e de PP com $50 \%$ de farinha de madeira seco ou após $48 \mathrm{~h}$ de imersão em água ${ }^{[1]}$.

Não se observa que haja grandes variações de rigidez entre os HIPS puros e nos compósitos, quando analisados com as mesmas quantidades de farinha de madeira. Apenas, nas amostras com $30 \%$ de farinha de madeira, pode-se dizer que o HIPS $495 \mathrm{~F}$ apresentou uma pequena redução no módulo de elasticidade em relação as demais, embora pouco significativa. Portanto neste caso, tanto o índice de fluidez quanto o tamanho das partículas de borracha não parecem ser determinantes para o módulo de elasticidade, embora na literatura se estabeleça que quanto maior o tamanho das partículas de borracha, menor deveria ser o módulo de elasticidade do HIPS ${ }^{[4]}$. 


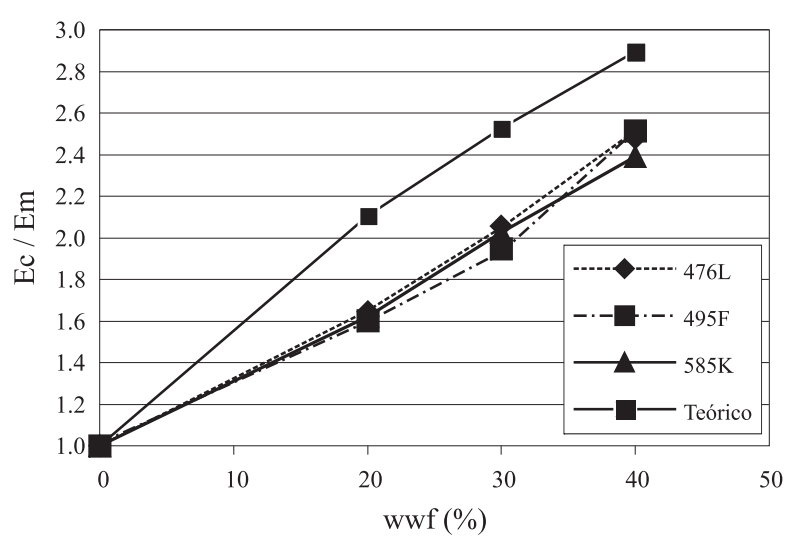

Figura 6. Razão entre módulos de elasticidade dos compósitos (Ec) e o módulo de elasticidade da matriz estirênica (Em) em função do teor de WWF no compósito.

Na Figura 6, são apresentados os valores de módulo relativo compósito/HIPS, ou seja, a razão dos módulos de elasticidade dos compósitos $\left(\mathrm{E}_{\mathrm{c}}\right)$ pelos módulos dos respectivos HIPS $\left(\mathrm{E}_{\mathrm{m}}\right)$ onde ficam evidentes os acréscimos de rigidez decorrentes da presença do reforço celulósico.

Observam-se acréscimos de cerca de $150 \%$ nos módulos das amostras com $40 \%$ de farinha de madeira, o que também tem-se verificado na literatura para compósitos de PP com $40 \%$ farinha de madeira ${ }^{[14-15]}$. Porém, o HIPS apresenta módulo de elasticidade à tração cerca de $25 \%$ superior ao $\mathrm{PP}^{[1]}$, podendo-se afirmar que o HIPS possui condições mais favoráveis de rigidez que o $\mathrm{PP}$, quando adicionados as mesmas quantidades do resíduo de farinha de madeira. As diferenças em módulos de elasticidade encontradas entre valores teóricos e determinadas nos ensaios dos compósitos podem ser relacionadas ao parâmetro de eficiência de reforço, K, conforme demonstrado a seguir.

\section{Cálculo do fator "K" como estimativa da adesão cargal matriz}

Aplicando a Regra das Misturas aos módulos elásticos, obtidos em laboratório para cada composição, pode-se determinar o fator " $\mathrm{K}$ ", a fim de avaliar indiretamente a eficiência de reforço celulósico ou adesão carga/matriz das

Tabela 7. Resultados do fator " $\mathrm{K}$ "

\begin{tabular}{lccc} 
Material & $\mathbf{4 7 6} \mathbf{L} \mathbf{2 0 w w f}$ & $\mathbf{4 9 5 F / 2 0 w w f}$ & $\mathbf{5 8 5 K} / \mathbf{2 0 w w f}$ \\
\hline *Ec (GPa) & 3,11 & 2,98 & 3,11 \\
$\mathbf{K}$ & 0,68 & 0,63 & 0,68 \\
\hline Material & $\mathbf{4 7 6 L / 3 0 w w f}$ & $\mathbf{4 9 5 F / 3 0 w w f}$ & $\mathbf{5 8 5 K / 3 0 w w f}$ \\
\hline *Ec (GPa) & 3,89 & 3,62 & 3,89 \\
$\mathbf{K}$ & 0,76 & 0,69 & 0,76 \\
\hline Material & $\mathbf{4 7 6 L / 4 0 w w f}$ & $\mathbf{4 9 5 F} / \mathbf{4 0 w w f}$ & $\mathbf{5 8 5 K} / \mathbf{4 0} \mathbf{w f}$ \\
\hline *Ec (GPa) & 4,69 & 4,68 & 4,59 \\
$\mathbf{K}$ & 0,82 & 0,82 & 0,80 \\
\hline
\end{tabular}

composições estudadas. Neste caso partiu-se de valores de módulos de elasticidade dos compósitos para cada composição obtidos experimentalmente conforme dados apresentados na Tabela 7 .

$$
{ }^{*} \mathrm{E}_{\mathrm{c}}=3,11=\mathrm{K} \times 8,5 \times 0,312+1,9 \times 0,688 \Rightarrow \mathrm{K}=0,68
$$

Os valores $\mathrm{K}$ obtidos para todas as demais misturas são apresentados na Tabela 7.

Os valores obtidos na Tabela 7 indicam que o fator " $\mathrm{K}$ " independe do tipo de resina mas aumenta com a concentração de carga no compósito. Nas composições com 20 e 30\% de resíduo, os dados sugerem uma possível melhor eficiência de reforço nas composições do HIPS 476L e 585K. No entanto nos compósitos com $40 \%$ de farinha de madeira, verificou-se não haver distinção entre o fator $\mathrm{K}$ calculado para as três resinas, uma vez que em concentrações elevadas o compósito 495F/ $40 \mathrm{wwf}$, praticamente, igualou-se às outras duas misturas. A eficiência da adesão na interface fibra-matriz deve estar associada a capacidade de molhamento da fibra pela resina, ou seja depende do índice de fluidez do material e da natureza química da interface. Todavia, embora o HIPS 495F devesse apresentar uma maior capacidade de molhamento da fibra devido ao seu índice de fluidez duas vezes superior as demais resinas, as análises da eficiência de reforço com base no Fator-K, não foram conclusivas para se avaliar diferenças de adesão em função do tipo de HIPS usado em cada compósito.

\section{Compósitos de fibra natural versus fibra de vidro}

A resistência específica $\left(\sigma_{\text {esp }}\right)$ e o módulo específico $\left(\mathrm{E}_{\text {esp }}\right)$ caracterizam o limite de resistência à tração em relação à densidade relativa, e o módulo de elasticidade em relação à densidade relativa, respectivamente.

Para efeito comparativo, usou-se a resistência e módulo específico do compósito HIPS com $35 \%$ de fibra de vidro e o valor calculado do compósito HIPS 476L/40\%wwf, que foi a mistura que apresentou maior resistência à tração e módulo de elasticidade neste trabalho. Os cálculos são descritos a seguir.

Cálculo $\rho_{\text {rel }}$ do compósito $476 \mathrm{~L} / 40 \% w w f$

$\rho_{\mathrm{c}}=(0,6 \times 1,05)+(0,4 \times 0,579)=0,86$ (densidade do compósito) onde: $\rho_{\mathrm{m}}=1.050 \mathrm{~kg} / \mathrm{m}^{3}$ (densidade da matriz) e $\rho_{\mathrm{f}}=579$ $\mathrm{kg} / \mathrm{m}^{3}$ (densidade da wwf)

$$
\begin{aligned}
& \text { Cálculo } \sigma_{\text {esp }} \text { e } \mathrm{E}_{\text {esp }} \text { do compósito } 476 \mathrm{~L} / 40 \% \mathrm{wwf} \\
& \sigma_{\text {esp }}=0,03 / 0,86=0,035 \mathrm{GPa} ; \\
& \mathrm{E}_{\text {esp }}=4,69 / 0,86=5,45 \mathrm{GPa} .
\end{aligned}
$$

Onde $\sigma_{\mathrm{m}}=0,03 \mathrm{GPa}$ (Tensão de escoamento da matriz, Figura 7)

Na avaliação dos compósitos HIPS com farinha de madeira e com fibra de vidro, observa-se que, mesmo com $5 \%$ de diferença de carga nesses compósitos, o material 476L/ $40 \%$ wwf apresentou resistência específica quase que três vezes superior a um compósito contendo fibra de vidro. Além 


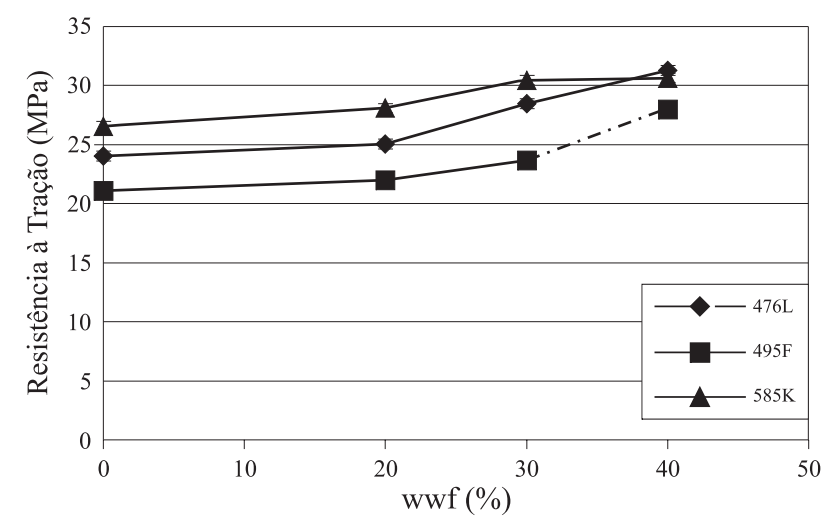

Figura 7. Efeito da concentração de madeira e do tipo de HIPS na Resistência à Tração no ponto de escoamento com Intervalo de Confiança de 95\%.

Tabela 8. Resistências e módulos específicos $\left(\sigma_{\mathrm{t}} \mathrm{e} \mathrm{E}_{\mathrm{t}}\right)$ comparativos para o HIPS reforçado com resíduo farinha de madeira e fibra de vidro.

\begin{tabular}{lccccc}
\hline \multicolumn{1}{c}{ Material } & $\begin{array}{c}\boldsymbol{\rho}_{\text {rel }} \\
\left(\mathbf{K g} / \mathbf{m}^{3}\right)\end{array}$ & $\begin{array}{c}\boldsymbol{\sigma}_{\mathbf{t}} \\
(\mathbf{G P a})\end{array}$ & $\begin{array}{c}\boldsymbol{\sigma}_{\text {esp }} \\
(\mathbf{G P a})\end{array}$ & $\begin{array}{c}\mathbf{E}_{\mathbf{t}} \\
(\mathbf{G P a})\end{array}$ & $\begin{array}{c}\mathbf{E} \\
(\mathbf{G P a})\end{array}$ \\
\hline $\begin{array}{l}\text { HIPS 525 com } \\
\text { 35\% fibra de vidro* }\end{array}$ & 1,59 & 0,02 & 0,013 & 2,1 & 1,3 \\
HIPS 476L/40\%wwf & 0,86 & 0,03 & 0,035 & 4,7 & 5,5 \\
\hline
\end{tabular}

* Valores obtidos da literatura ${ }^{[12]}$

disso, o módulo específico foi cerca de quatro vezes maior. Os resultados obtidos indicam condições de rigidez específica mais favoráveis de compósitos reforçados com farinha de madeira em relação à fibra de vidro.

$\mathrm{Na}$ prática, os efeitos de processamento produzem desfibrilamento e maior compactação da farinha de madeira no compósito. Ou seja, os produtos extrudados ou injetados a partir de compósitos termoplásticos celulósicos apresentam uma densidade real mais elevada do que os valores obtidos nos cálculos teóricos apresentados acima. Estes valores foram calculados a partir de dados da densidade aparente da farinha de madeira pré-processamento obtidos na literatura ${ }^{[8]}$. Valores da ordem de $1400 \mathrm{~kg} / \mathrm{m}^{3}$ para a farinha de madeira pós-processamento no compósito são encontrados na literatura o que de certa forma está em concordância com alguns aspectos relacionados no presente trabalho ${ }^{[14-16]}$.

Porém, embora seja competitiva em termos de propriedades específicas, deve-se destacar uma limitação da farinha de madeira em relação à fibra de vidro no que se refere à estabilidade térmica. A degradação da celulose ocorre na faixa de 200 a $220^{\circ} \mathrm{C}$ enquanto a fibra de vidro permanece estável nessa faixa de temperatura. Todavia, o uso de fibras naturais em compósitos termoplásticos apresenta menor desgaste de equipamentos e redução de impacto ambiental se comparado à fibra de vidro ${ }^{[17-18]}$.

\section{Resistência à Tração}

A Figura 7 ilustra a variação da resistência à tração no escoamento dos materiais compósitos em função da concentração de resíduo de madeira. Analisando as amostras de mesmo tipo de HIPS, verifica-se que o material puro apresenta uma menor resistência à tração, independente da concentração de farinha de madeira. Verifica-se que a incorporação de fibras eleva a resistência à tração de todos os compósitos em relação às suas matrizes, independente do teor de farinha de madeira. E quanto mais elevada a concentração de farinha de madeira, nessas misturas, observa-se que as tensões de escoamento elevam-se gradualmente. Observa-se um aumento da resistência à tração também no estudo do HIPS recuperado com $30 \%$ de farinha de madeira Pinus elliotti, porém em escala maior. Assim, pode-se dizer que a farinha de madeira eleva a resistência à tração, devido, provavelmente, a característica da estrutura química da celulose, cuja macromolécula possui fortes interações do tipo pontes de hidrogênio ${ }^{[19]}$. O Pinus caribea apresenta uma resistência à tração paralela às fibras de aproximadamente $65 \mathrm{MPa}^{[20]}$, quase três vezes maior que os HIPS puros, o que ajuda a compreender o aumento da resistência à tração quando adicionado farinha de madeira, caracterizado como reforço. Estudos mais aprofundados da adesão carga/polímero, quantidade de carga e características da carga e resina também devem ser considerados conforme previsto nos modelos de Halpin-Tsai, embora neste caso, a forma irregular das fibras dificulte a determinação dos parâmetros geométricos previstos no modelo ${ }^{[21]}$.

Na Figura 7 observam-se ainda dois aspectos importantes que dependem das características da resina. Os compósitos com resinas com índices de fluidez mais baixos (HIPS 585K e 476L) apresentaram uma tendência ao aumento da resistência à tração. Por outro lado, embora o tamanho de partículas seja determinante na tensão de escoamento dos materiais puros, esse efeito não foi observado nos compósitos. Nos casos estudados, a resistência à tração parece ser apenas uma função linear da concentração de madeira no compósito independentemente da fluidez e da FVPB.

\section{Tenacidade}

A capacidade do material absorver energia deformacional pode ser avaliada através dos ensaios de tração e impacto. Nas resinas puras, as características morfológicas e macromoleculares (tamanho médio de partícula e a FVPB) são determinantes no comportamento deformacional do HIPS $^{[5]}$. Todavia, nos compósitos a morfologia do HIPS torna-se irrelevante. Nos compósitos, tanto em tração como impacto observou-se uma redução drástica da tenacidade à

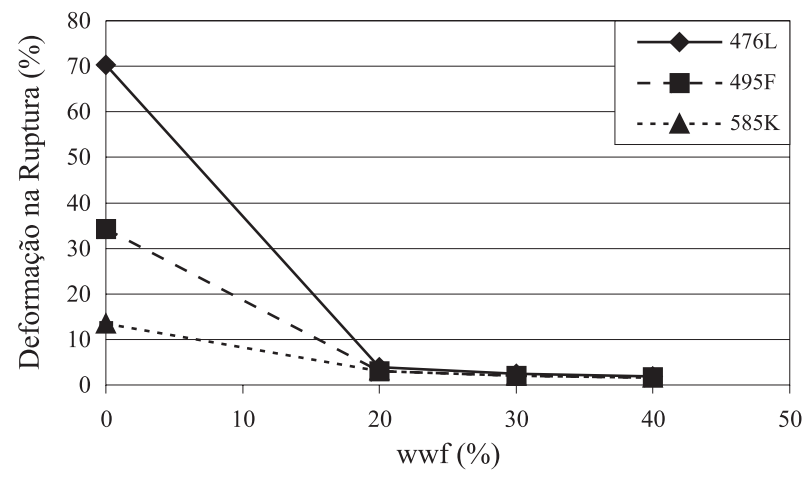

Figura 8. Efeito da concentração de madeira e do tipo de HIPS na Deformação na Ruptura em Tração com Intervalo de Confiança de 95\%. 


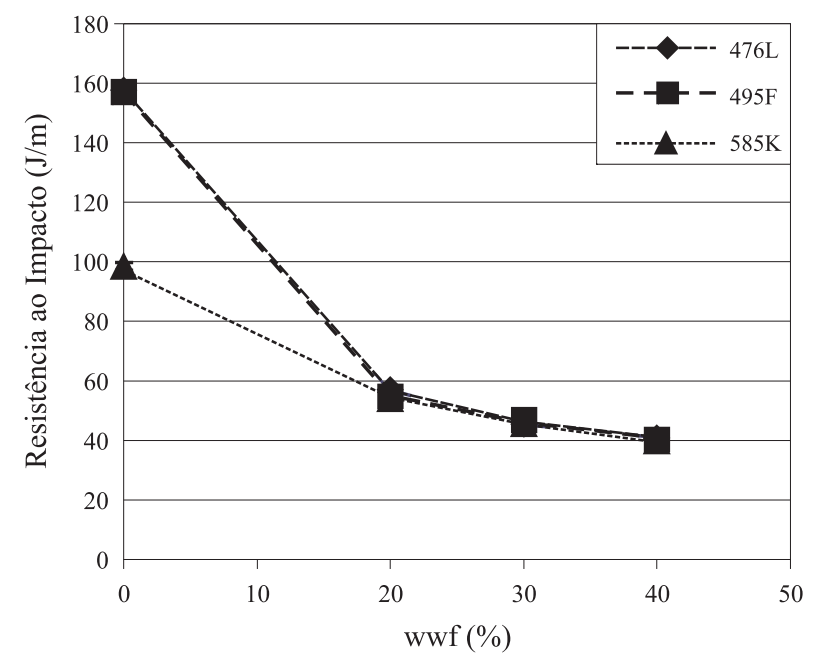

Figura 9. Efeito da concentração de farinha de resíduo de madeira e do tipo de HIPS na resistência ao impacto IZOD com intervalo de confiança de $95 \%$.

fratura com o aumento da concentração do reforço. Na Figura 8 são apresentadas as deformações na ruptura obtidas no ensaio de tração em função da concentração de carga para cada composição.

Observando-se o comportamento dos HIPS puros apresentados na Figura 8, verifica-se que o 476L possui uma maior deformação na ruptura do que o $495 \mathrm{~F}$ e $585 \mathrm{~K}$. Estes resultados confirmam que quanto maior o tamanho de partículas de borracha, maior a deformação na ruptura independente do índice de fluidez da resina. Portanto, embora possa haver outros aspectos não considerados, o principal fator de diferenciação da porcentagem de deformação na ruptura nos casos estudados é o tamanho médio das partículas de borracha.

No caso dos compósitos, apesar do aumento de rigidez específica, os resultados indicam que a presença do resíduo de madeira na matriz termoplástica inibe os mecanismos de tenacificação do material tornando-o relativamente frágil. De maneira geral, independente do tipo de matriz termoplástica, a adição de resíduo de madeira à termoplásticos tende a reduzir a porcentagem de deformação na ruptura, o que também foi observado em outros sistemas relacionados na literatura: PP com $40 \%$ de farinha de madeira ${ }^{[1]}$ e HIPS reciclado com $30 \%$ de farinha de madeira Pinus elliotti ${ }^{[13]}$.

O comportamento sob impacto do material apresenta características análogas ao apresentado sob tração conforme ilustrado na Figura 9 exceto pelas diferenças de tenacidade apresentadas pelas resinas puras. Sob altas taxas deformacionais o mecanismo de adesão carga-matriz tornase crítico uma vez que os mecanismos de reforço intrínsecos da resina tornam-se inoperantes na presença da carga.

\section{Temperatura de Distorção Térmica (HDT)}

Em se tratando de compósitos termoplásticos, a temperatura de distorção térmica é outro aspecto de grande interesse a ser avaliado. Os resultados dos ensaios de HDT apresentados na Figura 10 mostram que as concentrações do reforço celulósico contribuem significativamente para o aumento dessa propriedade elevando em até $10{ }^{\circ} \mathrm{C}$ a possibilidade de

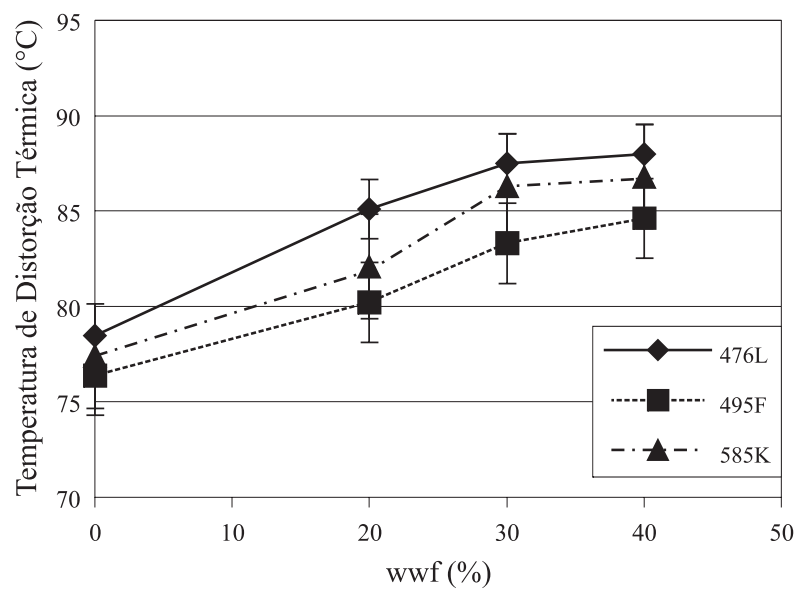

Figura 10. Efeito da concentração de resíduo de farinha de madeira e do tipo de HIPS na temperatura de distorção térmica (HDT) com intervalo de confiança de $95 \%$.

uso desses materiais.

\section{Considerações morfológicas}

Alguns aspectos morfológicos típicos dos compósitos estudados em relação a capacidade de dispersão do reforço na matriz e molhamento da fibra pela resina são apresentados na Figura 11 para compósitos de 495F, 476L e 585K contendo 20 e $40 \%$ de wwf. Nos dois primeiros casos são observados exemplos claros de delaminação na interface fibra-matriz e mecanismos de pull-out ou arrancamento da fibra durante a fratura. Estes mecanismos são característicos da deficiência de transferência de tensões na interface carga-matriz conforme indicado na micrografia. Os processos de delaminação poderiam explicar em parte as reduções observadas nos valores do Fator-K. Na maioria das composições, caracteriza-se bem o efeito de molhamento da carga pela resina, que devido a presença de anéis benzeno na cadeia do poli(estireno) poderiam estar favorecendo uma maior interação das cadeias poliméricas com a celulose ao contrário do comumente observado em matrizes poliolefinicas. Um melhor ancoramento das resinas estirênicas à superfície de cargas celulósica tem sido relacionada ao uso de agentes de acoplamento específicos à base isocianatos ${ }^{[22-24]}$. Estes produtos poderiam atenuar os processos de delaminação e pull-out e conseqüentemente aumentar a eficiência de reforço aproximando assim o Fator-K da unidade.

\section{Conclusões}

O HIPS é um termoplástico que pode ser utilizado em compósitos com madeira devido à facilidade de processamento a temperaturas abaixo da degradação da celulose e uma combinação de propriedades decorrentes da sua morfologia. Os compósitos produzidos apresentaram um bom desempenho mecânico embora melhorias no balanço de propriedades de rigidez e impacto se mostraram independentes da morfologia nas composições investigadas. Cálculos do módulo de elasticidade com base na Regra das Misturas para sistemas ideais aleatoriamente orientados possibilitaram uma avaliação indireta da eficiência de reforço e a rigidez do compósito pode ser 

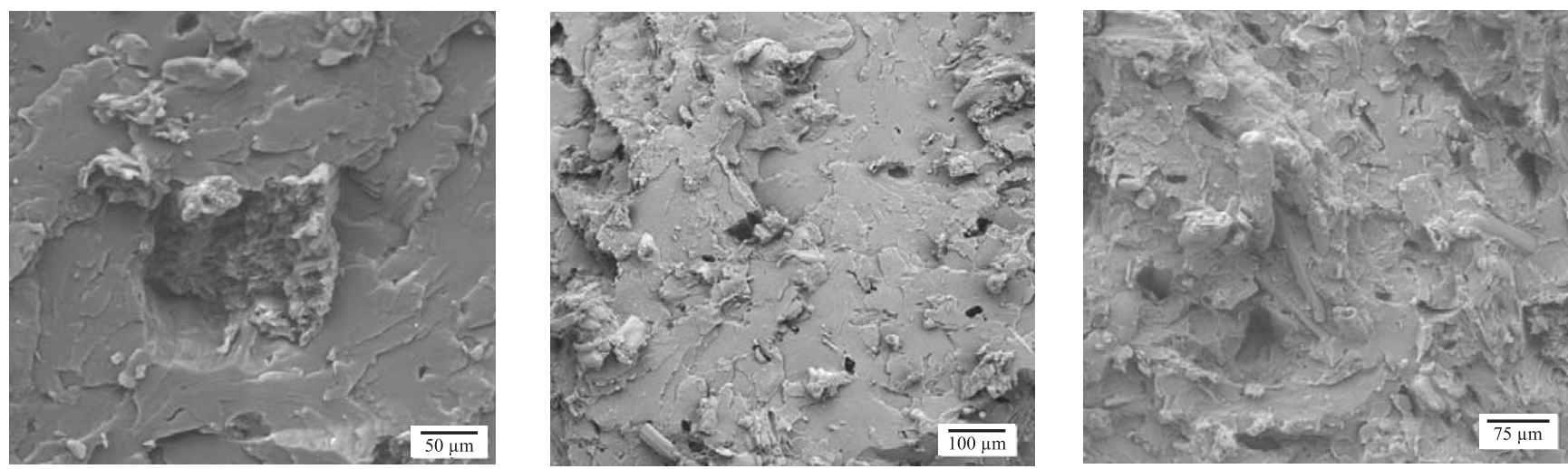

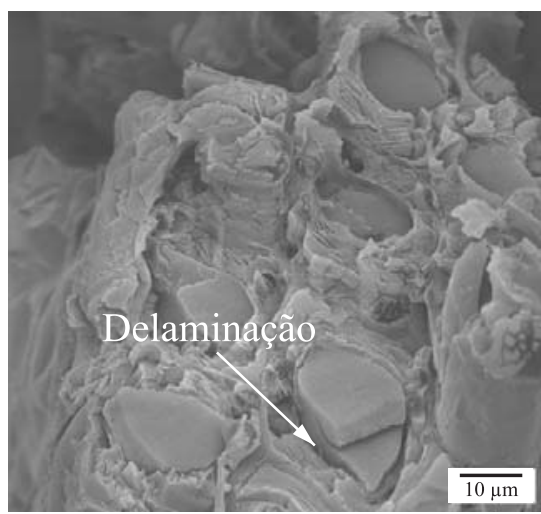

(a)

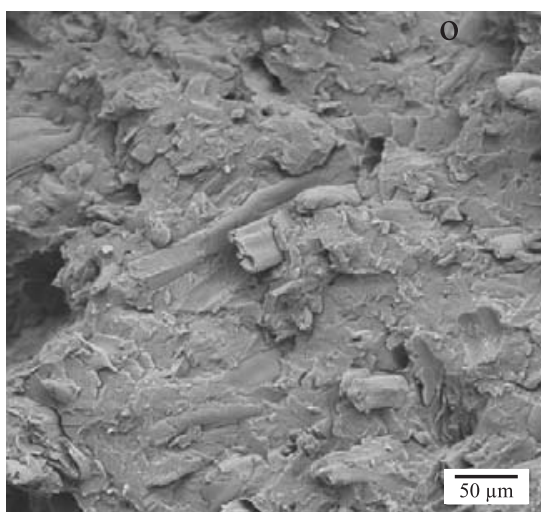

(b)

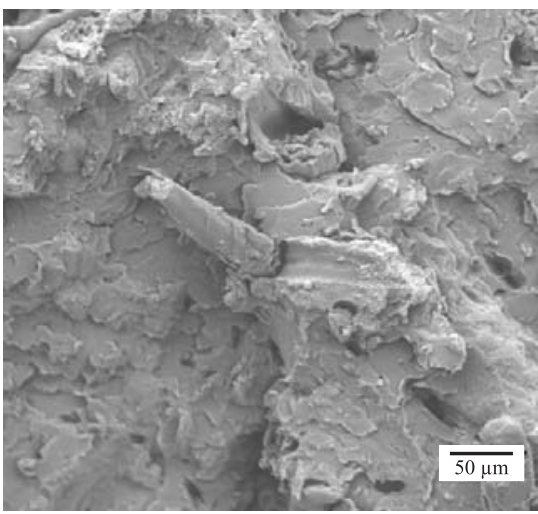

(c)

Figura 11. Aspectos morfológicos observados nas amostras (a) H495F/20 e 40WWF; (b) H476L/20 e 40WWF; (c) H585K 20 e 40WWF

prevista com base nas propriedades intrínsecas dos constituintes. Estudos mais apurados devem levar em consideração a densidade aparente do compósito (presença de vazios), decorrentes da liberação de voláteis no processamento, ou mesmo aspectos relacionados à morfologia da fibra celulósica que permitam a aplicação de modelos mais elaborados para determinação da rigidez em compósitos. Outro aspecto a ser explorado consiste na utilização de blendas de HIPS com copolímeros à base de SEBS (Estireno-Etileno-ButadienoEstireno) ou EPDM (Borracha de etileno-propileno) graftizados com anidrido maleico que possibilitem um melhor controle do nível de adesão fibra-matriz e particularmente da tenacificação da matriz termoplástica. No presente estudo, os teores máximos de borracha no material foram limitados pelo processo de obtenção do HIPS e a tenacidade dos materiais puros foi determinada pelo diâmetro médio e pela fração volumétrica das partículas de borracha (FVPB). No entanto, em todo os compósitos houve drástica redução da tenacidade com a adição do reforço celulósico independentemente da morfologia do HIPS utilizado como matriz no compósito.

Finalmente, através da simples aplicação da Regra das Misturas, verificou-se que devido à baixa densidade do resíduo de farinha de madeira, esta poderia substituir com vantagens outras cargas tradicionalmente utilizadas em termoplásticos. A incorporação de farinha de madeira ao HIPS apresentou excelente desempenho em termos de resistência e rigidez específica mostrando-se competitiva, em termos de custo-benefício, se comparada à fibra de vidro. Todavia a substituição de cargas minerais por reforços celulósicos ainda exige estudos mais aprofundados, sobretudo em relação à estabilidade térmica da celulose e durabilidade desses compósitos. Embora se mostre competitiva em termos de propriedades específicas, deve-se destacar a limitação da farinha de madeira em relação à fibra de vidro no que se refere à sua estabilidade térmica.

\section{Agradecimentos}

Os autores agradecem ao DEMa/UFSCar, a USF pela utilização dos laboratórios a CAPES pela bolsa de mestrado do aluno Wladimir Loesch Vianna e a FAPESP (Proc. 02/03909-5) pelo suporte financeiro.

\section{Referências Bibliográficas}

1. Correa, C.A.; Fonseca, C. N. P.; Neves, S.; Razzino, C.A. e Hage Jr,-- Compósitos Termoplásticos com Madeira. Polímeros: Ciência e Tecnologia, 3 (2003) p. 24-33.

2. Stark, N. e Scheneider, J.P.- Waste-Wood-Derived Fillers for Plastics, in: General Technical Report FPL-GTR91, Forest Product Laboratory/USDA, USA (1996).

3. Frollini, E.; Leão, A. L. e Mattoso, L. H. C. (Eds) - Natural Polymers and Agrofiber Based Composites, Embrapa Instrumentação Agropecuária, S.Carlos-SP, (2000). 
4. Echte, A. - Rubber Toughened Styrene Polymers: A Review in Rubber Toughened Plastics, Riew, C.K, Editor, p 15-64 (1989).

5. Grassi, V. G.; Forte, M. M. C.; Del Pizzol, M. F. - Aspectos Morfológicos e Relação Estrutura-Propriedades de Poliestireno de Alto Impacto. Polímeros: Ciência e Tecnologia. Vol. 11, nº3, p. 158-168, (2001).

6. Correa, C. A. - Caracterização de Polímeros Multifásicos. Parte 1: Processamento e Morfologia. Polímeros: Ciência e Tecnologia, Ano V, 1 p.154-165. São Carlos, SP (1995).

7. Catalogo Técnico de Resinas Plásticas da Empresa BASF S/A.

8. WEBSITE: www.set.eesc.usp.br/tarmem/ueda.htm

9. Bucknall, C. B.; Cote, F. F. P.; Partridge, I. K. - J. Mater. Sci. 21, 301 (1986).

10. Bucknall, C. B.; Davies, P.; Partridge, I. K. - J. Mater Sci. 22, 1341 (1987).

11. Maestrini, C.; Castellani, L.; Merlotti, M.; Vichi, M. - J. Mater. Sci. 27, 5994 (1993).

12. Callister Jr., W. D. - Materials Science and Engineering: An Introduction, $5^{\circ} \mathrm{Ed}$., Ch. 17, USA, (1999).

13. Desiderá, C. - Propriedades de compósitos de poliestireno de alto impacto (HIPS) proveniente de copos descartáveis pós-consumo e resíduos de serraria. (Dissertação de Mestrado em Ciência e Engenharia de Materiais) UFSCar, São Carlos, SP, (2002).

14. Yougquist, J.A. - Wood-Based Composites and Panel Products, Ch. 10, 1-31, (1999).

15. English, B. \& Clemons, C. M. - Weight reduction: wood versus mineral fillers in polypropylene, in: Proceedings of the Fourth International Conference on WoodfiberPlastic Composites, p. 237-244, Madison, Wisconsin, USA (1997).

16. English, B.; Clemons, C. M.; Stark, N. \& Scheneider, J. P. - Waste wood derived fillers for plastics. Technical Report, FPL/USDA (1996).

17. Wambua, P.; Ivens, J. \& Verpoest, I. - Natural Fibers: can they replace glass in fibre reinforced plastics?, Composite Science and Technology 63, p.1259-1264, (2003).

18. Joshi, S.V.; Drzal, L.T.; Mohanty, A.K. \& Arora, S. Are Natural Fiber Composites Environmentally Superior to Glass Fiber Reinforced Composites? Composites Part A In Press, (2003).

19. Fengel, D.; Wegener, G. - Wood: chemistry, ultrastructure, reactions, Berlim, Walter de Gruyter, (1984).

20. Revista da Madeira, No 73, Ano 13, (2003).

21. Hull, D. - An Introduction to Composite Materials, Cambridge University Press, (1981).

22. Maldas, D.; Kokta, B. V.; Deneault, C. - Influence of coupling agents and treatments on the mechanical properties of cellulose fiber-polystyrene composites. Journal of Applied Polymer Science, v.38, p.413-439, (1989).

23. Maldas, D.; Kokta, B. V. - Influence of phthalic anhydride as coupling agent on the mechanical properties of wood fiber-polystyrene composites. Journal of Applied Polymer Science, v.41, p.185-194, (1990).

24. Maldas, D.; Kokta, B. V. - Influence of maleic anhydride as coupling agent on the performance of wood fiberpolystyrene composites. Polymer Engineering and Science, v.31, n.18, p.1351-1357, (1991).

Enviado: 16/03/04

Reenviado: 08/07/04

Aprovado: 20/08/04 